\title{
Subjektives Alter und Weiterbildungsfunktionen. Zum Zusammenhang von Alter und Weiterbildungsteilnahme
}

\author{
Alexandra Maßmann (iD - Regina Egetenmeyer
}

Eingegangen: 20. Juli 2018 / Angenommen: 6. Dezember 2018 / Online publiziert: 18. Dezember 2018 (C) Der/die Autor(en) 2018

Zusammenfassung Der Beitrag analysiert den Zusammenhang des Alters von Weiterbildungsteilnehmenden und der von Teilnehmenden zugeschriebenen Funktion der Weiterbildungsteilnahme. Dazu wird der Einfluss des subjektiven Alters auf die Funktionszuschreibung einer Weiterbildung untersucht. Die Funktion wird nach beruflichen, persönlichen und sozialen Funktionen differenziert. Eine Faktorenanalyse legt eine zweifaktorielle Ausprägung des subjektiven Alters (kognitiv und sozialpsychisch) nahe. Mit Daten aus zwei Erhebungen ( $n=265$ und $n=441)$ erfolgt die Auswertung mittels Strukturgleichungsmodellen. Die Ergebnisse zeigen, dass das subjektive Alter keinen Zusammenhang zur beruflichen und persönlichen Funktion aufweist, mit steigendem sozial-psychischem subjektivem Alter die soziale Funktion aber abnimmt.

Schlüsselwörter Weiterbildungsforschung · Erwachsenenbildung · Funktion · Alter $\cdot$ Teilnahme $\cdot$ Lebensphasen

\section{Subjective age and the functions of continuing education. The linkage between age and participation in continuing education}

Abstract The article analyses the linkage between the age of participants of continuing education and the function which learners appoint to their participation.

Publisher's Note Springer Nature remains neutral with regard to jurisdictional claims in published maps and institutional affiliations.

Dr. A. Maßmann ( $₫)$

Bergische Universität Wuppertal, Wuppertal, Deutschland

E-Mail: massmann@uni-wuppertal.de

Prof. Dr. R. Egetenmeyer

Julius-Maximilians Universität Würzburg, Würzburg, Deutschland

E-Mail: regina.egetenmeyer@uni-wuerzburg.de 
Therefore, the influence of the subjective age for further education is studied. The study differentiates professional, social and personal functions of participation in continuing education. The result of a factor analysis designs subjective age into the two constructs cognitive and social-psychological subjective age. The data of two survey studies $(n=265$ and $n=441)$ are analysed with structural equation models. The results show, that the subjective age has no significant influence on the appointment to the professional and personal function. But, the analysis shows that an increasing subjective age is reducing the social function of continuing education.

Keywords continuing education research $\cdot$ adult education $\cdot$ function $\cdot$ age $\cdot$ participation · stages of life

\section{Einleitung}

Das Alter wurde in der Weiterbildungsforschung ${ }^{1}$ bisher vor allem als unabhängige Variable herangezogen und oftmals als Kontrollvariable untersucht. Die Relevanz des subjektiven Alters wurde bis jetzt hingegen wenig betrachtet.

Die Studie WeisA (Weiterbildungsteilnahme und subjektives Alter) untersucht die Einflüsse verschiedener Altersvariablen, speziell des subjektiven Alters, auf die Teilnahme an Weiterbildung. Insbesondere bei einer individuell berufsbezogenen oder nicht berufsbezogenen Teilnahme an Weiterbildung, ist von einem relativ hohen Maß an Freiwilligkeit der Teilnehmenden und daher von interindividuellen Unterschieden in den Teilnahmefunktionen auszugehen.

Es wird angenommen, dass eine Weiterbildungsteilnahme in Zusammenhang zum Alter der Teilnehmenden steht. Bisherige Studien zur Altersforschung in der Weiterbildung (z. B. Tippelt et al. 2009) beziehen sich vornehmlich auf das chronologische Alter. Die Altersforschung legt die Vermutung nahe, dass das chronologische Alter nur über eine eingeschränkte Erklärkraft verfügt (Staudinger 2012). Mit zunehmender Heterogenität von Lebensentwürfen und Selbstbildern bietet es sich deshalb an, das subjektive Alter auch zur Erklärung der Teilnahme an Weiterbildung heranzuziehen.

Der Beitrag geht zwei Forschungsfragen nach. Erstens: Welche Funktionen schreiben Erwachsene einer Weiterbildungsteilnahme zu? Zweitens: Welche Zusammenhänge zeigen sich zwischen dem (subjektiven) Alter und den Funktionen, die Teilnehmende einer Weiterbildung zuschreiben?

Zur Bearbeitung der Fragen, erfolgt zunächst die theoretische Begründung, die sich auf das Modell der Selektion, Optimierung und Kompensation stützt. Basierend auf den theoretischen Annahmen wurde eine Fragebogenstudie konzipiert, für die in zwei Erhebungsphasen Teilnehmende verschiedener Weiterbildungseinrichtungen in Bayern befragt wurden. Die Zusammenhänge, die sich zwischen den unabhängigen Altersvariablen sowie der beruflichen, persönlichen und sozialen Funktion ergeben, werden anhand inferenzstatistischer Ergebnisse erläutert. Die Diskussion

\footnotetext{
${ }^{1}$ Die Begriffe „Erwachsenenbildung“ und „Weiterbildung“ werden hier weitgehend synonym verstanden. Zur vereinfachen Lesbarkeit wird im Beitrag lediglich der Begriff „Weiterbildung“ verwendet.
} 
der Ergebnisse endet mit einem Ausblick auf die Relevanz der Ergebnisse für die Weiterbildungspraxis.

\section{Theoretische Begründung}

Die theoretische Grundlage der Studie liefert das Modell der selektiven Optimierung durch Kompensation (SOK-Modell) nach Baltes und Baltes (1989). Das Modell versucht, die Bedingungen erfolgreichen Alterns zu erklären. Die Autoren gehen von sechs Grundannahmen aus:

1. Man kann zwischen normalem, optimalem und krankem Altern unterscheiden.

2. Der Altersvorgang ist heterogen (variabel).

3. Es gibt eine beträchtliche „Stille Reserve“ (Plastizität).

4. In der Nähe der Leistungsreservegrenze gibt es einen altersbedingten Verlust.

5. Mit fortgeschrittenem Alter wird die Bilanz der Entwicklungsgewinne und -verluste zunehmend negativ.

6. Das Selbstbild bleibt auch im hohen Alter intakt (Baltes und Baltes 1989, S. 88 ff.).

Insbesondere die zweite Annahme, der heterogene Verlauf des Altersvorgangs, lässt vermuten, dass eine alleinige Erfassung des chronologischen Alters, als Summe der bereits abgelaufenen Lebensjahre, mit steigendem Alter eine immer geringere Erklärkraft aufweist (Staudinger 2012, S. 187). Dies begründet sich auch in der Annahme, dass das chronologische Alter eine Deckvariable darstellt (Staudinger 2012).

Die Funktion einer Weiterbildung ergibt sich aus den subjektiven Gründen für eine Teilnahme. In Anlehnung an von Hippel und Röbel (2016) werden Funktionen als „subjektive Bedeutungszuschreibungen“ (S. 64) an Weiterbildung verstanden. Die Funktionen umfassen somit die Aufgaben, die die Teilnahme an einer Weiterbildung im Leben einer Person erfüllt. Diese Gründe werden bei der Entscheidung für eine Kursanmeldung und auch bei der Anwendung des Gelernten relevant, die somit „bewusste, zielgerichtete Handlungen“ (Gorges und Kuper 2015, S. 2) darstellen. Die Funktionen einer Weiterbildung, die im Folgenden genauer zu betrachten sind, werden in berufliche, persönliche und soziale Funktion unterschieden. Es ist $\mathrm{zu}$ berücksichtigen, dass eine beruflich begründete Teilnahme nicht im Gegensatz zur persönlichen Funktion einer Teilnahme steht. In Anlehnung an die erneuerte Interessentheorie nach Grotlüschen (2010) kann ein ursprünglich berufliches Interesse zu einer zunächst unfreiwilligen Berührung mit einem Thema führen, woraus sich dann ein persönliches Interesse entwickeln kann. Die Funktionsbereiche schließen sich demnach nicht gegenseitig aus, können sich gegebenenfalls sogar gegenseitig bedingen.

Die Weiterbildungsforschung hat sich in Deutschland bereits bei einer ihrer ersten großen Studien, der Göttinger Studie, mit der Frage nach Funktionen und Gründen für eine Teilnahme beschäftigt (Strzelewicz et al. 1966). Auf die Frage nach dem Wunsch nach neuen Kontakten oder nach mehr Zeit allein berichteten $17 \%$ von einer sozialen Funktion ihrer Teilnahme (ebd., S. 89). Aktuelle Studien zeigen, dass insbesondere berufliche Gründe für eine Teilnahme häufig genannt werden (Beicht 
et al. 2004, S. 7). Mit dem Alter nimmt diese Funktion aber ab und persönliche und alltagsrelevante Gründe für eine Teilnahme treten deutlicher hervor (Schröder und Gilberg 2005; Kuwan et al. 2009). Es ist davon auszugehen, dass erwerbstätigen und damit tendenziell jüngeren Erwachsenen auch in stärkerem Maße betriebliche Weiterbildung zur Verfügung steht. In der vorliegenden Studie werden betriebliche Teilnahmegründe mit der beruflichen Funktion verknüpft.

Hinsichtlich des Zusammenhangs von Alter und Weiterbildung zeigt sich, dass Ältere seltener an Weiterbildung partizipieren. Nach Daten des Adult Education Survey (AES) nehmen die Gruppen der 65- bis 69-Jährigen sowie der 60- bis 64Jährigen am seltensten an Weiterbildung teil, die Gruppe der 35- bis 44-Jährigen am häufigsten (Bilger et al. 2017, S. 204). Dabei ist zu bedenken, dass die älteren Befragten bedingt durch Ruhestand seltener Zugang zu Erwerbstätigkeit und somit zu betrieblicher Weiterbildung haben. Auch die Volkshochschulstatistik verdeutlicht die seltenere Teilnahme Älterer. Noch weniger als diese nehmen aber junge Erwachsene unter 18 und zwischen 18 und 24 Jahren an Weiterbildung teil (Huntemann und Reichart 2017, S. 53). Dies ist mit den erhöhten Aktivitäten der Personen dieses Alters in anderen Bildungsbereichen (u.a. Hochschule, Berufsausbildung) zu erklären.

Der aktuelle Forschungsstand lässt die Frage nach dem Einfluss des subjektiven Alters der teilnehmenden Personen offen. Die meisten Studien hierzu befassen sich nur mit dem chronologischen Alter der Teilnehmenden. Forschungsergebnisse zu Entwicklungsprozessen zeigen jedoch (Staudinger 2012, S. 187), dass es sich beim chronologischen Alter um eine Deckvariable handelt. Das subjektive Alter beschreibt hingegen einen phänomenologischen Begriff und begründet sich im Selbst und in der Identität einer Person (Settersten und Godlewski 2016). Beim subjektiven Alter handelt es sich um eine persönlichere und differenziertere Einschätzung als es das chronologische Alter liefert (Thomae 1968). Eine Betrachtung der Funktionen von Weiterbildung vor dem Hintergrund des subjektiven Alters der Teilnehmenden erscheint auf dieser Grundlage erkenntnissteigernd.

Das subjektive Alter stellt, anders als das chronologische Alter, keine manifeste Variable dar. Die theoretischen Überlegungen der vorliegenden Arbeit legen eine Differenzierung des subjektiven Alters in die Bereiche kognitiv, sozial und psychisch nahe. Diese drei Bereiche ergeben sich in Anlehnung an die Differenzierung verschiedener Altersbereiche in Arbeiten von Kade (2009), Staudinger (2012) sowie Schmidt-Hertha und Mühlbauer (2012). Das kognitive Alter bezieht sich auf die persönliche Einschätzung der kognitiven Leistungsfähigkeit. Mit steigendem chronologischem Alter ist von einer nachlassenden kognitiven Leistung insbesondere im Bereich der fluiden Intelligenz auszugehen (Mietzel 2012; Schmitt 2004; Weinert 1994). Es ist anzunehmen, dass sich diese grundsätzliche Annahme der kognitiven Entwicklungen auch in der persönlichen Einschätzung widerspiegeln. Es wird daher angenommen, dass als nachlassend wahrgenommene kognitive Fähigkeiten tendenziell zu einer subjektiv älteren Einschätzung des empfundenen Alters führen, dies muss aber nicht so sein. Daher wird den Befragten auch keine genauere Definition des subjektiven Alters vorgegeben. Das soziale Alter geht mit einer Desintegration, nachlassenden sozialen Kontakten und rentenbedingtem Rollenverlust einher (Kade 2009; Künemann und Kohli 2010). Es ist dabei vor allem von Interesse, inwiefern 
Personen die sozialen Aspekte des Alters auch subjektiv wahrnehmen. Hierbei muss ein sozialer Rückzug nicht zwangsläufig zu einem höheren subjektiven Alter führen, relevant ist vielmehr die persönliche Einschätzung des eigenen sozialen Umfelds durch die Betreffenden. Zunehmendes psychisches Alter ist durch eine nachlassende psychische Spannkraft und Ausdauer zu charakterisieren (Weinert 1994; Kade 2009). Es ist davon auszugehen, dass mit zunehmender Desillusionierung, die das psychische Alter kennzeichnet, die Bereitschaft zur Aufnahme neuer Handlungen nachlässt, da unter anderem die Funktion der Handlungen für das eigene Leben nachlassen bzw. nicht mehr erkannt werden.

Bisherige empirische Untersuchungen zum subjektiv empfundenen Alter legen nahe, dass Personen sich mit steigendem chronologischem Alter subjektiv eher jünger einschätzen (Goldsmith und Heiens 1992; Rubin und Berntsen 2006). Ein solches niedrigeres subjektives Alter geht laut einer Studie von Teuscher (2009) darüber hinaus mit höherer Selbstwirksamkeit, Optimismus und Zufriedenheit mit dem eigenen Leben einher. Hieraus lässt sich die Vermutung ableiten, dass ein höheres subjektives Alter auf Seite der Befragten tendenziell negativ besetzt ist.

Dass das subjektiv empfundene Alter Einfluss auf die Einstellung und Präferenzen hat, konnte die Life-span-Forschung nach Carstensen zeigen (1995, 2006). Hierbei stellt sich heraus, dass Personen, die noch eine lange Lebenszeit erwarten, neue Informationen und Bekanntschaften anstreben. Personen, die nur noch eine kurze, überschaubare Lebenszeit erwarten, suchen eher Personen und Informationen auf, die einen emotionalen Mehrwert für sie haben (Carstensen 2006). Hieraus lässt sich ableitend annehmen, dass auch mit dem subjektiven Alter eine solch unterschiedliche Einschätzung von Situationen, zu denen auch Weiterbildung gehört, einhergeht.

In Anlehnung an das SOK-Modell und bisherige Ergebnisse zum subjektiven Alter werden folgenden Hypothesen aufgestellt:

$\mathbf{H}_{1 \mathbf{a}}$ Mit steigendem selbstperzipiertem kognitivem Alter nimmt die berufliche Funktion einer Weiterbildung ab.

$\mathbf{H}_{1 \mathrm{~b}}$ Mit steigendem selbstperzipiertem kognitivem Alter nimmt die persönliche Funktion einer Weiterbildung zu.

$\mathbf{H}_{2}$ Mit steigendem selbstperzipiertem sozialem Alter nimmt die soziale Funktion einer Weiterbildung zu.

$\mathbf{H}_{3 \mathbf{a}}$ Mit steigendem selbstperzipiertem psychischem Alter nimmt die berufliche Funktion einer Weiterbildung ab.

$\mathbf{H}_{3 \mathrm{~b}} \quad$ Mit steigendem selbstperzipiertem psychischem Alter nimmt die persönliche Funktion einer Weiterbildung ab.

$\mathbf{H}_{3 \mathbf{c}}$ Mit steigendem selbstperzipiertem psychischem Alter nimmt die soziale Funktion einer Weiterbildung ab. 


\section{Methode und Design}

Der Überprüfung der Hypothesen liegen zwei Erhebungen zugrunde, deren Daten zwischen Mai und Oktober 2015 erhoben wurden. Beide Studien sind querschnittlich angelegt, den Befragten wurde jeweils ein standardisierter Online-Fragebogen zur Verfügung gestellt. Bei den Befragten handelt es sich um Teilnehmende verschiedener Weiterbildungsveranstaltungen. Die erfassten Items und Konstrukte sind für beide Studien im Wortlaut gleich. Hierdurch wird sichergestellt, dass sich die Ergebnisse beider Studien vergleichen lassen.

\subsection{Stichproben}

Für die erste Erhebung, im Folgenden als Erhebung Fernkurs bezeichnet, wurden Teilnehmende des Kurses „Theologie im Fernkurs - Grundkurs Theologie“, organisiert von der Domschule Würzburg, befragt. Die Daten wurden bereinigt, sodass nur Personen, die mindestens 30\% des Fragebogens bearbeitet haben, im Datensatz enthalten sind. Nach der Bereinigung umfasst die Stichprobe von Erhebung Fernkurs 265 Personen. Diese Erhebung umfasst eine stark selektive Stichprobe, da Teilnehmende eines konfessionell orientierten Fernkurses befragt wurden. Aus diesem Grund und auf Basis der Erfahrungen aus der Erhebung Fernkurs, wurde eine zweite Studie durchgeführt. Hierzu wurden Teilnehmende verschiedener Kurse an Volkshochschulen (VHS) und Industrie- und Handelskammern (IHK) in Bayern befragt. Die im Folgenden als Erhebung VHS/IHK bezeichnete Erhebung umfasst nach der Bereinigung des Datensatzes 441 Fälle.

Die Befragten in Erhebung Fernkurs sind zwischen 22 bis 74 Jahre alt $(M W=46,44$; $S D=10,08)$. Die Stichprobe setzt sich aus 51,6\% männlichen und 48,4\% weiblichen Befragten zusammen. Als höchsten Schulabschluss geben die meisten Personen das (Fach-)Abitur an (72,65\%), 54,67\% haben einen (Fach-)Hochschulabschluss. Darüber hinaus verfügen $57,21 \%$ der Befragten über einen akademischen Abschluss und erwerbstätig sind 76,68\%.

Die Erhebung VHS/IHK umfasst Personen zwischen 19 und 85 Jahren, im Mittel liegt das Alter bei 44,79 Jahren $(S D=14,52)$. Diese Stichprobe umfasst mit $68,83 \%$ deutlich mehr Frauen. Hier ist der häufigste Schulabschluss die Mittlere Reife/Realschulabschluss mit 46,78\%, dicht gefolgt vom Abitur (43,81\%). 32,65\% der Befragten haben einen akademischen Abschluss erworben und auch hier sind mit 76,37\% über ein Drittel der Befragten erwerbstätig.

Hinsichtlich des Alters unterscheiden sich die Studien nicht deutlich voneinander. Durch die stärkere Beteiligung von Frauen an Kursen der VHS (Huntemann und Reichart 2017) lässt sich der Anteil von Teilnehmerinnen in Erhebung VHS/IHK in Ansätzen erklären. Es ist in der Erhebung VHS/IHK außerdem anzunehmen, dass IHKs durch ihr umfangreiches Angebot in der Aufstiegsfortbildung im Anschluss an eine Berufsausbildung vor allem Personen mit mittleren Schulabschlüssen als Zielgruppe ansprechen. 


\subsection{Operationalisierung}

Um das Alter zu erfassen werden das chronologische sowie das subjektive Alter herangezogen. Das chronologische Alter wird mittels einer offenen numerischen Angabe erhoben. Beim subjektiven Alter handelt es sich um eine latente Variable. Die Befragten sollten hierzu eine Selbsteinschätzung ihres subjektiv empfundenen Alters vornehmen. Dabei wurde Ihnen keine Definition des subjektiven Alters als positive oder negative Entwicklung vorgegeben. Die Einschätzung der Bedeutung eines höheren oder niedrigeren subjektiven Alters obliegt somit den Befragten selbst. Das subjektive Alter wurde in den Erhebungen differenziert in die Bereiche kognitives, soziales und psychisches subjektives Alter. Hierzu wurden jeweils drei Items entwickelt (Bsp.-Items: kognitiv Aufmerksamkeitsspanne; sozial Teilnahme an gesellschaftlichen Ereignissen; psychisch Seelische Belastbarkeit). Die abhängige Variable Funktion wird in drei Bereichen mittels einer vierstufigen Skala (1 = stimmt überhaupt nicht bis $4=$ stimmt völlig) erfasst. Das Konstrukt berufliche Funktion (Bsp.-Items: Ich nehme an dem Kurs teil, um meinen beruflichen Alltag besser ausüben zu können.) umfasst neun Items. Der Skalenmittelwert liegt jeweils unter dem theoretischen Skalenmittelwert (Erhebung Fernkurs: $M=2,0 ; S D=0,78$; Erhebung VHS/IHK: $M=2,19 ; S D=1,05)$. Die Reliabilität ist jeweils als gut zu bewerten (Erhebung Fernkurs: $\alpha=0,90$; Erhebung VHS/IHK: $\alpha=0,96$ ).

Das Konstrukt soziale Funktion (Bsp.-Item: Ich nehme an dem Kurs teil, um in einer Gruppe mit anderen zu lernen.) umfasst sechs Items. Die Skalenmittelwerte liegen unter dem theoretischen Mittel (Erhebung Fernkurs: $M=2,18 ; S D=0,70$; Erhebung VHS/IHK: $M=2,37 ; S D=0,71)$. Das Konstrukt ist in beiden Studien als reliabel einzuschätzen (Erhebung Fernkurs: $\alpha=0,85$; Erhebung VHS/IHK: $\alpha=0,86$ ).

Die persönliche Funktion (Bsp.-Item: Ich nehme an dem Kurs teil, um mich persönlich herauszufordern.) umfasst ebenfalls sechs Items. Die Skalenmittelwerte liegen mit $M=3,29(S D=0,51)$ in Erhebung Fernkurs und $M=3,24(S D=0,53)$ in Erhebung VHS/IHK über dem theoretischen Skalenmittel. Die Reliabilität des Konstrukts ist gegeben (Erhebung Fernkurs: $\alpha=0,77$; Erhebung VHS/IHK: $\alpha=0,79$ ).

Für das kognitive subjektive Alter liegen die Mittelwerte in beiden Studien unter den Mittelwerten des jeweiligen chronologischen Alters (Erhebung Fernkurs: $M=40,82 ; S D=11,86$; Erhebung VHS/IHK: $M=39,41 ; S D=13,01)$. Die Skala erweist sich in beiden Studien als reliabel (Erhebung Fernkurs: $\alpha=0,91$; Erhebung VHS/IHK: $\alpha=0,93$ ).

Das sozial-psychische subjektive Alter zeigt in beiden Studien etwas niedrigere Mittelwerte als das kognitive subjektive Alter (Erhebung Fernkurs: $M=40$; $S D=9,76$; Erhebung VHS/IHK: $M=38,72 ; S D=11,73$ ). Auch hier ist die Reliabilität in beiden Studien als gegeben einzuschätzen (Erhebung Fernkurs: $\alpha=0,82$; Erhebung VHS/IHK: $\alpha=0,84$ ). Zur Überprüfung der (konkurrenten) Kriteriumsvalidität (Bühner 2011, S. 63) der neu entwickelten Skala zum subjektiven Alter wurden in der Erhebung Fernkurs zusätzlich Daten anhand der Nürnberger-Selbsteinschätzungs-Liste (NSL) nach Oswald und Fleischmann (1999) erhoben. Die NSL umfasst eine vierstufige Antwortskala ( $1=$ trifft nicht $z u$ bis $4=$ trifft $z u$, Bsp.-Item: „Es fällt mir in letzter Zeit schwerer, mich mit Problemen auseinanderzusetzen"). Die Reliabilität der Skala erweist sich mit einem Cronbachs $\alpha$ von 0,93 als gegeben. Die 
Korrelationen zeigen, dass die NSL sowohl mit dem subjektiven sozial-psychischen Alter $(r=0,30 ; p<0,001)$, als auch mit dem subjektiven kognitiven Alter $(r=0,42$; $p<0,001)$ signifikant zusammenhängt.

Zusätzlich zu diesen unabhängigen und abhängigen Variablen wird der Einfluss weiterer möglicher Variablen kontrolliert. Hierzu werden Erwerbs- und Bildungsstatus sowie Geschlecht herangezogen und als Dummy-Variablen kodiert (Erwerbsstatus $1=$ erwerbstätig, $0=$ nicht erwerbstätig; Bildungsstatus $1=$ akademischer Berufsabschluss; 0=nicht-akademischer Berufsabschluss; Geschlecht 1=weiblich, $0=$ männlich).

\subsection{Zur Datenanalyse}

Die vorliegenden Daten werden in mehreren Schritten ausgewertet. Neu konstruierte Skalen werden mit explorativen Faktorenanalysen (Bortz und Schuster 2010; Döring und Bortz 2016) kontrolliert. Die Anzahl zu extrahierender Faktoren wird anhand des Kaiser-Guttmann-Kriteriums festgelegt (Döring und Bortz 2016, S. 482). Danach erfolgt die Faktorenanalyse mittels orthogonaler Varimax-Rotation, um eine inhaltliche Interpretation der Faktoren zu ermöglichen (Werner 2014).

Die sich hierdurch ergebenen latenten Variablen werden im Anschluss mithilfe einer Reliabilitätsanalyse (Cronbach 1951) auf ihre interne Konsistenz überprüft. Die Ergebnisse dieser Auswertungsschritte wurden bereits unter 3.2 Operationalisierung berichtet.

Für die inferenzstatistische Hypothesenprüfung wird das Gesamtmodell im Querschnitt mittels Strukturgleichungsmodellen untersucht. Diese werden mithilfe der Software Mplus 6.0 berechnet (Muthén und Muthén 2010). Hierbei wird die in Mplus voreingestellte Full Information Maximum Likelihood (FIML)-Schätzung für fehlende Werte genutzt (Lüdtke et al. 2007, S. 112; Geiser 2010, S. 33).

Die Möglichkeit, anhand des Messmodells eine konfirmatorische Faktorenanalyse in Mplus durchzuführen, besteht mit den vorliegenden Daten nicht, da hier nicht genügend Fälle für die einzelnen benötigten Variablen vorhanden sind.

Aufgrund der Vielzahl zu überprüfender Variablen wird das theoretische Modell nicht komplett untersucht, sondern in kleinere Strukturgleichungsmodelle (berufliche, soziale und persönliche Funktion) aufgeteilt.

Die Modellpassung der Strukturgleichungsmodelle ist Tab. $1 \mathrm{zu}$ entnehmen (Geiser 2010; Reinecke und Pöge 2010; Schermelleh-Engel et al. 2003).

Tab. 1 Statistische Tests und Indizes zur Beurteilung der Modellpassung der Strukturgleichungsmodelle

\begin{tabular}{lllllll}
\hline & Studie & RMSEA & SRMR & CFI & TLI & Chi $^{2}$ \\
\hline Funktion - beruflich & A & 0,162 & 0,056 & 0,809 & 0,591 & Signifikant \\
& B & 0,157 & 0,043 & 0,885 & 0,755 & Signifikant \\
Funktion - sozial & A & 0,017 & 0,025 & 0,998 & 0,993 & Nicht signifikant \\
& B & 0,000 & 0,012 & 1,000 & 1,016 & Nicht signifikant \\
& A & 0,162 & 0,056 & 0,778 & 0,525 & Signifikant \\
& B & 0,157 & 0,044 & 0,857 & 0,693 & Signifikant \\
\hline
\end{tabular}


Die Werte der Modellgüte (vgl. Tab. 1) machen deutlich, dass die Güte der Modelle Funktion - beruflich sowie Funktion - persönlich nicht durch alle Indizes bestätigt ist. Ein hoher Messfehler kann daher nicht ausgeschlossen werden.

Dies ist darauf zurückzuführen, dass diese Modelle über eine unabhängige Variable mehr verfügen, als das Modell Funktion - sozial. Dadurch werden weitere Pfade möglich, die aber vor dem theoretischen Hintergrund nicht hinreichend sinnvoll in das Modell aufzunehmen sind. Eine mögliche eingeschränkte Aussagekraft muss daher bei der Interpretation dieser Modelle beachtet werden. Eine vorsichtige Interpretation der Werte wird aber dennoch vorgenommen, da vor allem die Werte des SRMR nur geringfügig vom Richtwert abweichen.

Die verringerte Reliabilität der Daten und teilweise eingeschränkte Güte der Strukturgleichungsmodelle in den genannten Fällen wirkt sich auf die Zuverlässigkeit der Ergebnisse aus. Es ist bei der Betrachtung und Interpretation zu berücksichtigen, dass der Messfehler in den genannten Bereichen über dem akzeptierten Wert liegt und die Ergebnisse somit nicht ausreichend gegen den Zufall abgesichert sind.

\section{Zusammenhang Alter und Funktion}

Im Folgenden werden die Ergebnisse der Strukturgleichungsmodelle berichtet. Aufgrund der sehr unterschiedlich zusammengesetzten Stichproben erfolgt die Berechnung für die Erhebungen Fernkurs und VHS/IHK getrennt. Um die Lesbarkeit und Vergleichbarkeit zu erleichtern, werden die Ergebnisse beider Erhebungen aber in jeweils einer Darstellung visualisiert.

Die Ergebnisse der explorativen Faktorenanalyse der Studie legen nahe, dass das subjektive Alter nur zwei Faktoren, das kognitive und das sozial-psychische subjektive Alter, umfasst. Alle Hypothesen, die sich ursprünglich auf das soziale bzw. psychische subjektive Alter beziehen, umfassen nun das sozial-psychische Alter als erklärende Variable. Die neuen und nun jeweils zu testenden Hypothesen werden im Folgenden nochmal aufgeführt.

\subsection{Berufliche Funktion}

Auf Basis des SOK-Modells (Baltes und Baltes 1989) wird angenommen, dass die berufliche Teilnahme an Weiterbildung in der Regel der Optimierung von Ressourcen dient und somit vor allem im jungen und mittleren Erwachsenenalter eine hohe Bedeutung hat. Es ist daher davon auszugehen, dass die Relevanz beruflicher Funktion mit dem Alter, unter anderem auch rentenbedingt, zurückgeht.

Zum Zusammenhang von subjektivem Alter und beruflicher Funktion werden demnach folgende Hypothesen geprüft:

$\mathbf{H}_{1 \mathbf{a}}$ Mit steigendem selbstperzipiertem kognitivem Alter nimmt die berufliche Funktion einer Weiterbildung ab. 


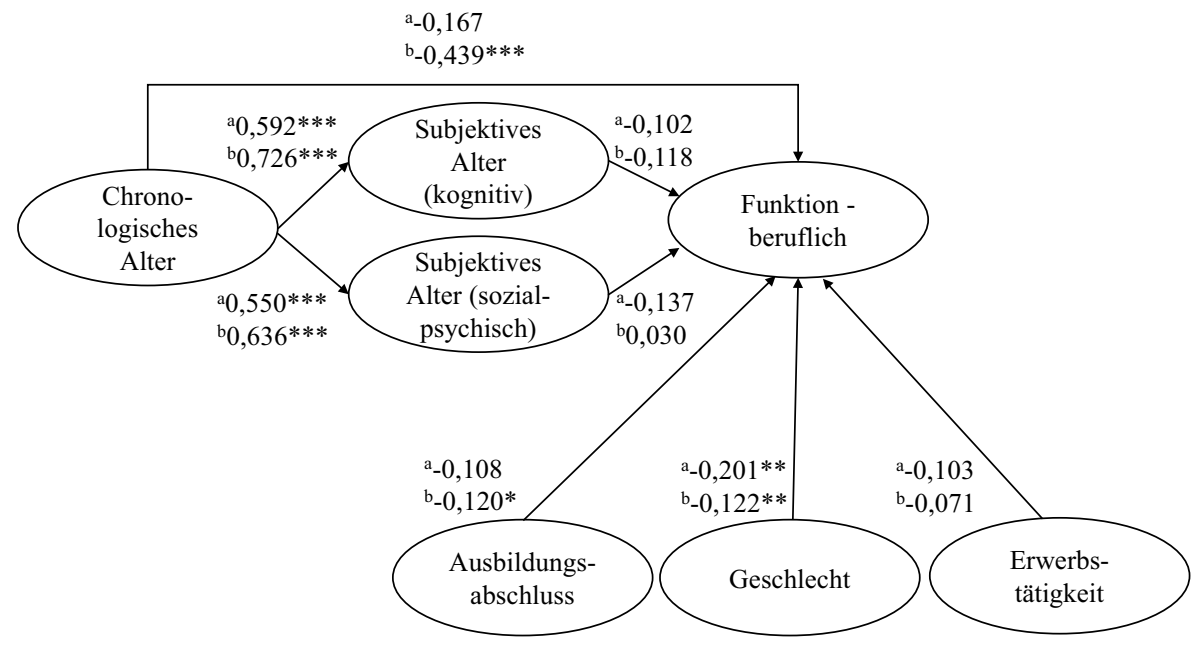

Abb. 1 Strukturgleichungsmodell zum Zusammenhang von Alter und beruflicher Funktion ( ${ }^{a}$ Erhebung Fernkurs; ${ }^{b}$ Erhebung VHS/IHK; $* p \leq 0,05 ; * * p \leq 0,01 ; * * * p \leq 0,001$ )

$\mathbf{H}_{3 \mathbf{a}} \quad$ Mit steigendem selbstperzipiertem sozial-psychischem Alter nimmt die berufliche Funktion einer Weiterbildung ab.

Die Berechnungen des Pfadmodells zeigen für die Erhebung Fernkurs neben einer signifikant geringeren Ausprägung beruflicher Funktion von Frauen gegenüber Männern keinen weiteren signifikanten Zusammenhang mit der beruflichen Funktion. In der Erhebung VHS/IHK bestätigt sich der negative Zusammenhang des chronologischen Alters und der beruflichen Funktion. Die berufliche Funktion scheint demnach deutlicher vom chronologischen als vom subjektiven Alter bestimmt (Abb. 1). Dies lässt sich in Ansätzen dadurch erklären, dass der berufliche Lebenslauf relativ stark an das chronologische Alter gebunden ist. Die Aufnahme einer Erwerbstätigkeit und die damit zuvor einhergehende berufliche Qualifizierung in Form von Ausbildung, Studium und Weiterbildung folgen einer Chronologie deutlicher als einer subjektiven Einstellung. Besonders deutlich wird dies etwa am Renteneintrittsalter, welches chronologisch festgelegt ist. Demnach liegt es nahe, dass auch die berufliche Bedeutung von Weiterbildung dieser Chronologie folgt.

$\mathrm{H}_{1 \mathrm{a}}$ sowie $\mathrm{H}_{3 \mathrm{a}}$ müssen daher auf Grundlage der Ergebnisse aus beiden Erhebungen abgelehnt werden.

\subsection{Persönliche Funktion}

Im Zusammenhang zur Annahme der nachlassenden beruflichen Funktion, ist davon auszugehen, dass mit steigendem subjektivem Alter die Bedeutung der persönlichen Funktion einer Teilnahme dementsprechend zunimmt. Aus dem SOK-Modell lässt sich hierzu die Annahme ableiten, dass wegfallende berufliche Gründe und Optionen für Weiterbildung mit zunehmendem Alter durch die Fokussierung auf persönliche Interessen kompensiert werden, die dann unter anderem in der Weiter- 
bildung verfolgt werden. Vormals berufliche Teilnahmegründe können dabei auch ein persönliches Interesse an einem Thema generiert haben (Grotlüschen 2010). Mit steigendem Alter und insbesondere in der Nacherwerbsphase werden diese Themen dann aus rein persönlichen Gründen verfolgt. Daneben ist zu berücksichtigen, dass mit steigendem psychischem subjektivem Alter von einem generellen Nachlassen der Funktionen einer Weiterbildung auszugehen ist, da hiermit die Motivation in Form einer psychischen Spannkraft (Kade 2009) nachlässt. Es gilt demnach folgende Hypothesen zu prüfen:

$\mathbf{H}_{1 \mathrm{~b}} \quad$ Mit steigendem selbstperzipiertem kognitivem Alter nimmt die persönliche Funktion einer Weiterbildung zu.

$\mathbf{H}_{\mathbf{3 b}} \quad$ Mit steigendem selbstperzipiertem sozial-psychischem Alter nimmt die persönliche Funktion einer Weiterbildung ab.

Die Erhebung Fernkurs weist keinerlei Zusammenhänge zwischen persönlicher Funktion und Alter auf. Aufgrund der konfessionell gebundenen Kursthematik kann angenommen werden, dass Teilnehmende dieser Veranstaltung altersunabhängig einen persönlichen Mehrwert in der Teilnahme sehen.

Für die Erhebung VHS/IHK zeigt das Strukturmodell einen positiven Zusammenhang des chronologischen Alters und der persönlichen Funktion. Es zeigt sich im Vergleich der beruflichen und persönlichen Funktion auch, dass zumindest in Studie B mit dem chronologischen Alter die berufliche Funktion ab- und die persönliche Funktion zunimmt (Abb. 2). Dies war auf Basis des Forschungsstandes und der theoretischen Überlegungen so zu erwarten und ist mit den Ergebnissen bisheriger Forschung konform. Da sich zwischen den subjektiven Altersvariablen und der

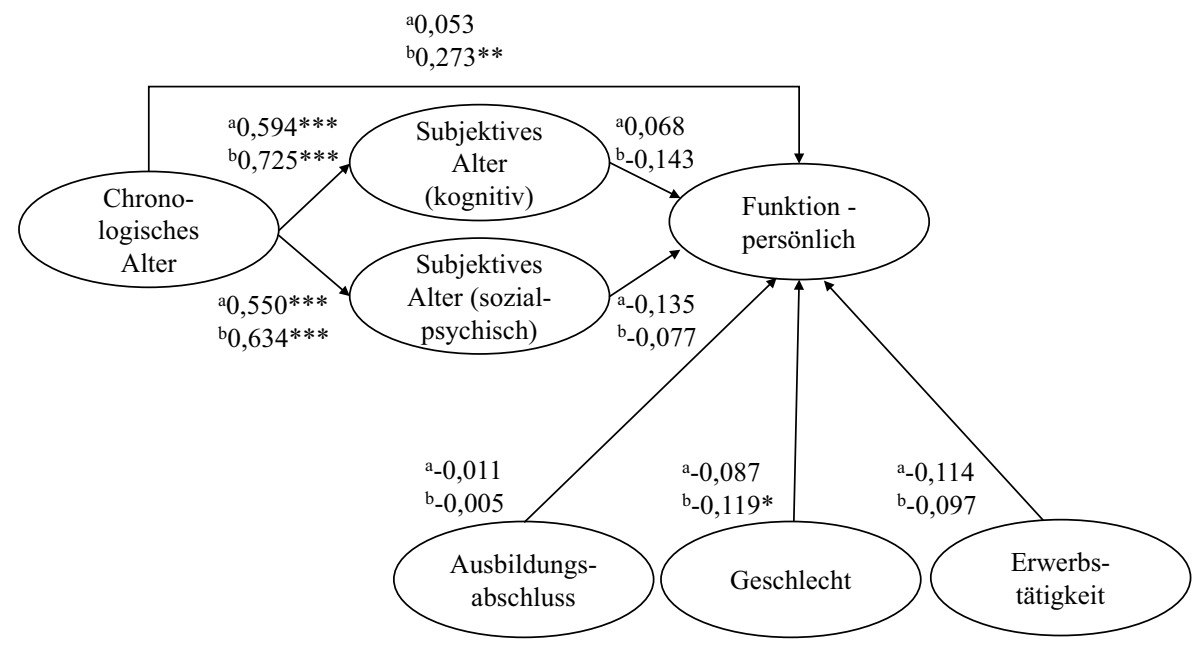

Abb. 2 Strukturgleichungsmodell zum Zusammenhang von Alter und persönlicher Funktion ('Erhebung Fernkurs; ${ }^{b}$ Erhebung VHS/IHK; ${ }^{*} p \leq 0,05 ; * * p \leq 0,01 ; * * * p \leq 0,001$ ) 
persönlichen Funktion die konstatierten Zusammenhänge nicht zeigen, sind $\mathrm{H}_{1 \mathrm{~b}}$ und $\mathrm{H}_{3 \mathrm{~b}}$ abzulehnen.

\subsection{Soziale Funktion}

Mit steigendem Alter kommt es zu einem Rückgang sozialer Kontakte, verursacht durch soziale Desintegration und einhergehend mit Faktoren wie dem Ausstieg aus dem Erwerbsleben (Kade 2009; Künemann und Kohli 2010). Eine Weiterbildungsteilnahme stellt in der Regel eine soziale Situation dar, die mit verschiedenen Interaktionen einhergeht. Eine Teilnahme kann, in Anlehnung an das SOK-Modell, dazu dienen, wegfallende soziale Kontakte durch den Austausch mit anderen und das gemeinsame Lernen in der Gruppe zu kompensieren. Auch dies ist vermutlich eine mit steigendem Alter zunehmend bedeutsamer werdende Funktion von Weiterbildung. Aufgrund der Ergebnisse der explorativen Faktorenanalyse umfasst das subjektive Alter nun, wie bereits berichtet, nur noch zwei Faktoren. Dies führt dazu, dass $\mathrm{H}_{2}$ und $\mathrm{H}_{3 \mathrm{c}}$ nun gegenteilige Annahmen machen. Bei der Auswertung gilt es zu klären ob und wenn ja, welche der beiden Hypothesen zutrifft:

$\mathbf{H}_{2}$ Mit steigendem selbstperzipiertem sozial-psychischem Alter nimmt die soziale Funktion einer Weiterbildung zu.

$\mathbf{H}_{3 \mathbf{c}}$ Mit steigendem selbstperzipiertem sozial-psychischem Alter nimmt die soziale Funktion einer Weiterbildung ab.

Die Ergebnisse verdeutlichen, dass chronologisch Ältere eine höhere soziale Funktion in Weiterbildungsveranstaltungen wahrnehmen als chronologisch Jüngere. Des Weiteren geht mit steigendem sozial-psychischem subjektivem Alter eine Abkehr von der sozialen Funktion vonstatten. Wer sich sozial-psychisch älter fühlt, sieht in einer Weiterbildungsveranstaltung weniger die Möglichkeit neuer sozialer Kontakte und des Austausches. Die Erhebung Fernkurs zeigt eine höhere soziale Funktion für Personen ohne akademischen Abschluss. In der Erhebung VHS/IHK wird deutlich, dass Erwerbstätige weniger soziale Funktion in Veranstaltungen sehen als Nicht-Erwerbstätige. Dies deutet möglicherweise darauf hin, dass die Erwerbstätigkeit soziale Funktionen übernimmt. Insgesamt verdeutlichen die Ergebnisse zur sozialen Funktion den bedeutsamen und unterschiedlichen Einfluss der verschiedenen Altersvariablen auf die Teilnahme aus sozialen Gründen (Abb. 3). Stärker als berufliche und persönliche Funktion scheint die soziale Funktion mit dem Alter einhergehenden Veränderungen zu unterliegen. Mit steigendem chronologischem Alter erfolgt eine Weiterbildungsteilnahme stärker aus sozialen und weniger aus beruflichen Gründen. $\mathrm{H}_{3 \mathrm{c}}$ ist somit abzulehnen, aber $\mathrm{H}_{2}$ trifft laut der Ergebnisse beider Erhebungen zu und kann somit vorläufig angenommen werden. Es ist hierbei besonders zu beachten, dass sich die Bedeutung des subjektiven und chronologischen Alters für die soziale Funktion in beiden Erhebungen, das heißt sowohl in klassischen Präsenzkursen (Erhebung VHS/IHK), als auch in einem Fernkurs-Setting mit einem kleineren Anteil an Präsenzveranstaltungen (Erhebung Fernkurs) zeigt. Es lässt sich daher vermuten, dass nicht primär das Ausmaß an Austausch relevant 


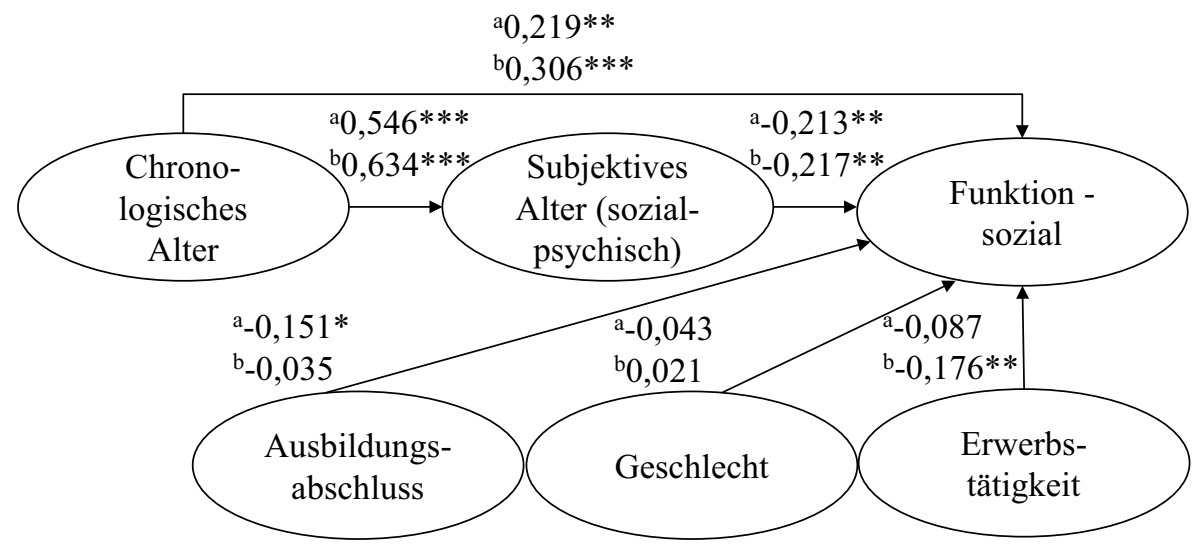

Abb. 3 Strukturgleichungsmodell zum Zusammenhang von Alter und sozialer Funktion ( ${ }^{\mathrm{a} E r h e b u n g ~ A ; ~}$ ${ }^{\mathrm{b}}$ Erhebung VHS/IHK; $\left.* p \leq 0,05 ; * * p \leq 0,01 ; * * * p \leq 0,001\right)$

für die soziale Funktion ist, sondern die grundsätzliche Möglichkeit zum sozialen Austausch, auch wenn dieser seltener stattfindet.

\section{Diskussion und Ausblick}

Die eingangs benannten Forschungsfragen sind anhand der Studienergebnisse wie folgt zu beantworten:

1. Welche Funktionen schreiben Erwachsene einer Weiterbildungsteilnahme zu? Weiterbildungsteilnahmen aus vorrangig individuell berufsbezogenen oder nicht berufsbezogenen Gründen, gehen deutlich mit einer persönlichen Funktion für die Teilnehmenden einher. Dies zeigt sich in beiden Erhebungen anhand der Mittelwerte von 3,29 (Fernkurs) bzw. 3,24 (VHS/IHK). Es folgen die soziale Funktion mit mittleren Zustimmungswerten von 2,18 (Fernkurs) und 2,37 (VHS/IHK) und zuletzt die berufliche Funktion mit Mittelwerten von 2,0 (Fernkurs) und 2,19 (VHS/IHK).

2. Welche Zusammenhänge zeigen sich zwischen dem (subjektiven) Alter und den Funktionen, die Teilnehmende einer Weiterbildung zuschreiben? Die Ergebnisse verdeutlichen die Relevanz des chronologischen Alters für die berufliche Funktion. In der Erhebung VHS/IHK zeigt sich mit steigendem chronologischem Alter eine signifikante Abnahme der beruflichen Funktion der Weiterbildung. Aufgrund des chronologischen Aufbaus beruflicher Biographien und den mit dem Alter und der Nacherwerbsphase nachlassenden beruflichen Gründen und Verwertungszusammenhängen von Kursinhalten, entspricht dieses Ergebnis den bisherigen Befunden der Weiterbildungsforschung. Auf dieser Grundlage lässt sich auch erklären, dass das subjektive Alter keine zusätzlichen Erklärungen liefert. Der nicht signifikante Zusammenhang des chronologischen Alters und der beruflichen Funkti- 
on in der Erhebung Fernkurs deutet aber an, dass dieser Zusammenhang nicht in jedem Kurssetting vorhanden sein muss.

Ein sehr ähnliches Bild zeigt sich bei der Betrachtung der persönlichen Funktion. Diese steigt in der Erhebung VHS/IHK mit dem chronologischen Alter an. Die aus dem SOK-Modell abgeleitete Vermutung, dass wegfallende berufliche Ressourcen und Lernbedarfe im Alter in Form der Verfolgung persönlicher Interessen in Weiterbildung kompensiert werden, lässt sich hieran erkennen. Aber auch die persönliche Funktion zeigt in der Erhebung Fernkurs keinen Zusammenhang zum chronologischen Alter und insgesamt keinen Zusammenhang zu den subjektiven Altersvariablen.

In deutlichem Zusammenhang zum Alter steht die soziale Funktion. Diese nimmt in beiden Erhebungen mit dem chronologischen Alter zu und mit dem sozial-psychischen subjektiven Alter ab. Hieran wird deutlich, dass das subjektive Alter eine eigenständige Erklärkraft aufweist und neben dem chronologischen Alter Betrachtung finden sollte.

Neben den bereits erwähnten thematischen und didaktischen Engführungen der Erhebung Fernkurs ist die Geschlechteraufteilung in der Erhebung VHS/IHK kritisch zu erwähnen. Die Aufteilung ist weniger ausgeglichen als in der Erhebung Fernkurs. Hier finden sich deutlich mehr Frauen, vermutlich bedingt durch die generell stärkere Teilnahme von Frauen bei Angeboten der Volkshochschulen (Huntemann und Reichart 2017). Verzerrungen der Ergebnisse durch die Selektivität der einzelnen Stichproben sind nicht auszuschließen. Auch die Ergebnisse der inferenzstatistischen Auswertung weisen vor allem auf die Kontextabhängigkeit der Befunde zur beruflichen und persönlichen Funktion hin. Aussagen lassen sich demnach nur für einen eingeschränkten Teil von Weiterbildungsangeboten machen. Vor allem zu betrieblicher Weiterbildung sowie informell organisierten Lernsettings können auf Grundlage der vorliegenden Daten keine Aussagen gemacht werden. Darüber hinaus beziehen die vorliegenden Stichproben keine Personen ein, die zum Zeitpunkt der Befragung nicht an Weiterbildung teilnehmen.

Trotz der angeführten Einschränkungen lässt sich aus der Studie eine Bedeutung des subjektiven Alters, insbesondere für die soziale Funktion der Weiterbildung, erkennen. Die Erfassung des subjektiven neben dem chronologischen Alter bietet dabei die grundsätzliche Möglichkeit, Stichproben genauer zu beschreiben. Die Berücksichtigung des subjektiven Alters, vor allem bei der Betrachtung sozialer Aspekte von Weiterbildung, kann einen Mehrwert bei der Betrachtung bestimmter Zielgruppen und Adressatinnen und Adressaten liefern. Aufgrund der eigenständigen Erklärkraft, die dem subjektiven neben dem chronologischen Alter demnach zukommt, bietet sich eine Weiterentwicklung dieses komplexen Konstrukts an, um es für weitere Forschung und Fragestellungen nutzbar zu machen. Grundlage dieser Überarbeitung kann die Forschung zu Altersselbstbildern bieten. Dabei ist davon auszugehen, dass nicht nur die Einschätzung eines aktuellen subjektiven Alters Relevanz für die Teilnahme und Wahrnehmung einer Weiterbildung hat, sondern insbesondere zukünftige Erwartungen an das eigene Alter bei der Planung und Einschätzung von Veranstaltungen relevant erscheinen. Arbeiten hierzu konnten bereits zeigen, dass negative Altersselbstbilder zu der Annahme führen, keinen positiven 
Einfluss auf das eigene Alter nehmen zu können (Schmidt-Hertha und Mühlbauer 2012; Staudinger 2012). Dies, so lässt sich vermuten, nimmt demnach auch essentiellen Einfluss auf die Teilnahme an und Einschätzung von Weiterbildung. Darüber hinaus bleibt zu untersuchen, wie sich das subjektive Alter in Weiterbildungen für bestimmte Zielgruppen, wie z. B. Seniorenkursen, darstellt. Im Zusammenhang mit betrieblicher Weiterbildung stellt sich des Weiteren die Frage, inwiefern das subjektive Alter bzw. Altersselbstbild und der Übergang in die Nacherwerbsphase in Zusammenhang stehen. Die Bedeutung des subjektiven Alters für betriebliche sowie informelle Lernsettings stellen somit noch offene Forschungsansätze dar. Auch die Erforschung der Bedeutung des empfundenen Alters für das Fernbleiben von Weiterbildung lässt sich an die hier vorliegenden Ergebnisse anschließen.

Die vorgestellten Ergebnisse verweisen auf eine besondere Rolle der sozialen Funktion, die auch für die Praxis der Weiterbildung Relevanz besitzt. Da die soziale Funktion auch in Hinblick auf unterschiedliche Kurssettings mit steigendem chronologischen Alter an Bedeutung gewinnt, erscheint die Betonung sozialer und gemeinschaftlicher Aspekte des Kurses vor allem für chronologisch Ältere von besonderer Bedeutung. Subjektiv Ältere und chronologisch Jüngere hingegen besuchen einen Kurs tendenziell weniger aus sozialen Gründen. Dies ist bei der Auswahl didaktischer Arrangements sowie bei der Wahrnehmung von Widerständen gegenüber bestimmten Seminarmethoden zu berücksichtigen.

Open Access Dieser Artikel wird unter der Creative Commons Namensnennung 4.0 International Lizenz (http://creativecommons.org/licenses/by/4.0/deed.de) veröffentlicht, welche die Nutzung, Vervielfältigung, Bearbeitung, Verbreitung und Wiedergabe in jeglichem Medium und Format erlaubt, sofern Sie den/die ursprünglichen Autor(en) und die Quelle ordnungsgemäß nennen, einen Link zur Creative Commons Lizenz beifügen und angeben, ob Änderungen vorgenommen wurden.

\section{Literatur}

\section{Verwendete Literatur}

Baltes, P. B., \& Baltes, M. M. (1989). Optimierung durch Selektion und Kompensation - Ein psychologisches Modell erfolgreichen Alterns. Zeitschrift für Pädagogik, 35, 85-105.

Beicht, U., Krekel, E.M., \& Walden, G. (2004). Berufliche Weiterbildung - welchen Nutzen haben die Teilnehmer? Berufsbildung in Wissenschaft und Praxis, 5, 5-9.

Bilger, F., Behringer, F., Kuper, H., \& Schrader, J. (2017). Weiterbildungsverhalten in Deutschland 2016. Ergebnisse des Adult Education Survey (AES). Bielefeld: W. Bertelsmann.

Bortz, J., \& Schuster, C. (2010). Statistik für Human- und Sozialwissenschaftler (7. Aufl.). Berlin, Heidelberg: Springer.

Bühner, M. (2011). Einführung in die Test- und Fragebogenkonstruktion (3. Aufl.). München: Pearson Studium.

Carstensen, L. L. (1995). Evidence for a life-span theory of socioemotional selectivity. Current directions in psychologigal science, 4(5), 151-156.

Carstensen, L.L. (2006). The influence of a sense of time on human development. Science, 312, 1913-1915.

Cronbach, L. J. (1951). Coefficient alpha and the internal structure of tests. Psychometrika, 16, 297-334.

Döring, N., \& Bortz, J. (2016). Datenerhebung. In N. Döring \& J. Bortz (Hrsg.), Forschungsmethoden und Evaluation in den Sozial- und Humanwissenschaften (5. Aufl. S. 321-577). Berlin, Heidelberg: Springer.

Geiser, C. (2010). Datenanalyse mit Mplus. Eine anwendungsorientierte Einführung (2. Aufl.). Wiesbaden: VS. 
Goldsmith, R.E., \& Heiens, R. A. (1992). Subjective age: a test of five hypotheses. The Gerontologist, 32(3), 312-317.

Gorges, J., \& Kuper, H. (2015). Editorial. Motivationsforschung im Weiterbildungskontext. Zeitschrift für Erziehungswissenschaft, 18, 1-7.

Grotlüschen, A. (2010). Erneuerung der Interessetheorie. Die Genese von Interesse an Erwachsenen- und Weiterbildung. Wiesbaden: VS.

von Hippel, A., \& Röbel, T. (2016). Funktionen als aktuersabhängige Zuschreibung in der Programmplanung betrieblicher Weiterbildung. Report Zeitschrift für Weiterbildungsforschung, 39(1), 61-81.

Huntemann, H., \& Reichart, E. (2017). Volkshochschul-Statistik. 55. Folge, Arbeitsjahr 2016. Bielefeld: W. Bertelsmann.

Kade, S. (2009). Altern und Bildung: Eine Einführung (2. Aufl.). Bielefeld: W. Bertelsmann.

Künemann, H., \& Kohli, M. (2010). Soziale Netzwerke. In K. Aner \& U. Karl (Hrsg.), Handbuch Soziale Arbeit und Alter (S. 309-313). Wiesbaden: VS.

Kuwan, H., Tippelt, R., \& Schmidt, B. (2009). Weiterbildungs-Erwartungen, Bildungsbarrieren und Informationsbedarf. In R. Tippelt, B. Schmidt, S. Schnurr, S. Sinner \& C. Theisen (Hrsg.), Bildung Älterer - Chancen im demografischen Wandel (S. 156-172). Bielefeld: W. Bertelsmann.

Lüdtke, O., Robitzsch, A., Trautwein, U., \& Köller, O. (2007). Umgang mit fehlenden Werten in der psychologischen Forschung. Probleme und Lösungen. Psychologische Rundschau, 58(2), 103-117.

Mietzel, G. (2012). Entwicklung im Erwachsenenalter. Göttingen: Hogrefe.

Muthén, L. K., \& Muthén, B. O. (2010). Mplus 6.0. Los Angeles: Muthén \& Muthén. Computer software

Oswald, W.D. \& Fleischmann, U.M. (1999). Nürnberger Alters-Inventar: (NAI) (4. Aufl.). Göttingen: Hogrefe.

Reinecke, J., \& Pöge, A. (2010). Strukturgleichungsmodelle. In C. Wolf \& H. Best (Hrsg.), Handbuch der sozialwissenschftlichen Datenanalyse (S. 775-804). Wiesbaden: VS.

Rubin, D.C., \& Berntsen, D. (2006). People over forty feel $20 \%$ younger than their age: subjective age across the lifespan. Psychonomic Bulletin \& Review, 13(5), 776-780.

Schermelleh-Engel, K., Moosbrugger, H., \& Müller, H. (2003). Evaluating the fit of structural equation models: tests of significance and descriptive goodness-of-fit measures. Methods of Psychological Research Online, 8(2), 23-74.

Schmidt-Hertha, B., \& Mühlbauer, C. (2012). Lebensbedingungen, Lebensstile und Altersbilder älterer Erwachsener. In F. Berner, J. Rossow \& K.-P. Schwitzer (Hrsg.), Individuelle und kulturelle Altersbilder. Expertisen zum Sechsten Altenbericht der Bundesregierung, (Bd. 1, S. 109-149). Wiesbaden: VS.

Schmitt, E. (2004). Aktives Altern, Leistungseinbußen, soziale Ungleichheit und Altersbilder. Ein Beitrag zum Verständnis von Resilienz und Vulnerabilität im höheren Erwachsenenalter. Zeitschrift für Gerontologie und Geriatrie, 37, 280-292.

Schröder, H., \& Gilberg, R. (2005). Weiterbildung Älterer im demographischen Wandel. Empirische Bestandsaufnahme und Prognose. Bielefeld: W. Bertelsmann.

Settersten, R. A., \& Godlewski, B. (2016). Concepts and theories of age and aging. In V.L. Bengtson \& R. A. Settersten (Hrsg.), Handbook of theories of aging (S. 9-25). New York: Springer.

Staudinger, U.M. (2012). Fremd- und Selbstbild im Alter. Innen- und Außensicht und einige der Konsequenzen. In P. Graf Kielmansegg \& H. Häfner (Hrsg.), Alter und Altern. Wirklichkeit und Deutung (S. 187-200). Berlin, Heidelberg: Schneider.

Strzelewicz, W., Schulenberg, W., \& Raapke, H.-D. (1966). Bildung und gesellschaftliches Bewußtsein. Stuttgart: Enke.

Teuscher, U. (2009). Subjective age bias: a motivational and information processing approach. International Journal of Behavioral Development, 33(1), 22-31.

Thomae, H. (1968). Zur Entwicklungs- und Sozialpsychologie des alternden Menschen. In H. Thomae \& U. Lehr (Hrsg.), Altern. Probleme und Tatsachen (S. 3-17). Frankfurt a. M.: Akademische Verlagsgesellschaft.

Tippelt, R., Schmidt, B., Schnurr, Sinner, S., \& Theisen, C. (2009). Bildung Älterer. Chancen im demografischen Wandel. Bielefeld: W. Bertelsmann.

Weinert, F.E. (1994). Altern in psychologischer Perspektive. In P. B. Baltes, J. Mittelstraß \& U. M. Staudinger (Hrsg.), Alter und Altern: Ein interdisziplinärer Studientext zur Gerontologie (S. 180-203). Berlin: de Gruyter.

Werner, C. (2014). Explorative Faktorenanalyse: Einführung und Analyse mit R. Universität Zürich. http://www.psychologie.uzh.ch/fachrichtungen/methoden/team/christinawerner/faktorenanalyse/ explorative_faktorenanalyse_mit_r_cswerner.pdf. Zugegriffen: 16. Febr. 2017. 


\section{Weiterführende Literatur}

Maßmann, A. (2018). Einflussfaktor subjektives Alter - Effekte auf die Teilnahme an und die Bewertung von Weiterbildungen. Bielefeld: W. Bertelsmann. 